\title{
Effect of Glycerol on Blood Flow Distribution in Tumoral and Peritumoral Brain Tissue
}

\author{
Masahiro NIWA, Hirofumi OYAMA, Tatsuya KOBAYASHI, \\ Yoshihisa KIDA, Takayuki TANAKA, Satoshi MAESAWA, \\ Masahiro FURUSE*, Suguru INAO**, \\ and Jun YosHIDA**
}

\begin{abstract}
Department of Neurosurgery, Komaki City Hospital, Komaki, Aichi; *Department of Neurosurgery, Nakatsugawa Municipal General Hospital, Nakatsugawa, Gifu; ** Department of Neurosurgery, Nagoya University School of Medicine, Nagoya
\end{abstract}

\begin{abstract}
The effect of glycerol on blood flow in tumoral and peritumoral tissue was measured in 32 patients with brain tumor, 17 gliomas and 15 meningiomas. Blood flow before and after the administration of glycerol was measured by stable xenon-enhanced computed tomography. The tumor part of glioma was significantly hypoperfused. In contrast, the tumor part of meningioma was significantly hyperperfused. Peritumoral edema of both glioma and meningioma was hypoperfused. After the administration of glycerol, blood flow increased in all regions except for the tumor part of glioma. Vascular responses to glycerol may be different in these two tumor types. The steal phenomena of blood flow might occur in cases of glioma.
\end{abstract}

Key words: glycerol, blood flow, glioma, meningioma

\section{Introduction}

Glycerol causes reduction of brain edema by osmotic difference, and improves blood flow. Glycerol is used to treat patients with brain tumor, but the effect of glycerol on blood flow in such patients has not been well studied. This study measured blood flow in tumors and peritumoral edema to evaluate the effect of glycerol intravenous administration on blood flow, and evaluated the difference in flow distribution between glioma and meningioma.

\section{Materials and Methods}

Thirty-two patients, 21 males and 11 females aged from 15 to 75 years (mean 45.9 years), were treated for 17 gliomas (15 glioblastomas, 2 astrocytomas) and 15 meningiomas (13 meningothelial, 2 malignant). The diagnosis was confirmed by histological examination of surgical specimens.

Blood flow was measured by stable xenon-en- hanced computed tomography (Xe-CT) during inhalation of $25 \%$ stable xenon for 3 minutes and washout for 5 minutes. Regions of interest (ROIs) were selected in the tumor, the peritumoral edema, and the control value (contralateral brain corresponding to the edema). The hemispheric blood flow on the tumor side and the non-tumor side were also measured. ROIs were designed by free hand according to the tumor shape, by reference to CT scans with contrast media and the $T_{1}$-weighted magnetic resonance (MR) images with gadolinium-diethylenetriaminepenta-acetic acid (Gd-DTPA) for the tumor, and CT scans and $T_{2}$-weighted MR images for the peritumoral edema. The tumor was defined as the lesion enhanced by the contrast media and the peritumoral edema as the high intensity lesion on the $\mathrm{T}_{2}$-weighted $\mathrm{MR}$ image. The mean diameter of the tumor was $25 \mathrm{~mm}$ for gliomas and $39 \mathrm{~mm}$ for meningiomas. The maximum distance of the peritumoral edema from the tumor margin, on the $\mathrm{T}_{2}$-weighted MR images, was $28 \mathrm{~mm}$ for glioma and

Received February 14, 1997; Accepted August 4, 1997

Author's present address: M. Niwa, M.D., Department of Neurosurgery, Nakatsugawa Municipal General Hospital, Nakatsugawa, Gifu, Japan. 
Table 1 Cerebral blood flow in various brain regions of patients with tumor treated with glycerol

\begin{tabular}{|c|c|c|c|c|c|c|}
\hline & \multicolumn{3}{|c|}{ Glioma } & \multicolumn{3}{|c|}{ Meningioma } \\
\hline & Before & After & After/Before & Before & After & After/Before \\
\hline Tumor & $36.4 \pm 5.2^{*}$ & $31.6 \pm 5.2^{*}$ & $86.9 \pm 7.3^{\#}$ & $73.7 \pm 6.8^{*}$ & $80.4 \pm 6.8^{*}$ & $109.2 \pm 7.4$ \\
\hline Peritumoral edema & $37.5 \pm 4.8^{*}$ & $44.6 \pm 4.8^{*}$ & $119.7 \pm 5.4$ & $33.8 \pm 5.3^{*}$ & $36.7 \pm 4.4^{*}$ & $108.8 \pm 6.9$ \\
\hline Control region & $55.6 \pm 4.2$ & $69.5 \pm 3.3$ & $125.1 \pm 8.7$ & $54.0 \pm 6.2$ & $65.8 \pm 6.3$ & $121.9 \pm 6.3$ \\
\hline Tumor hemisphere & $52.1 \pm 7.1$ & $58.2 \pm 5.4$ & $111.8 \pm 6.0$ & $53.5 \pm 4.1$ & $58.5 \pm 5.5$ & $109.5 \pm 7.2$ \\
\hline Non-tumor hemisphere & $56.9 \pm 4.4$ & $66.1 \pm 4.5$ & $116.3 \pm 7.6$ & $52.4 \pm 4.7$ & $60.8 \pm 4.2$ & $116.1 \pm 9.3$ \\
\hline
\end{tabular}

Values are mean $\pm \mathrm{SD}$ blood flow in $\mathrm{ml} / 100 \mathrm{~g} / \mathrm{min}$ before and after glycerol administration, and percentage of blood flow after/before treatment with glycerol. ${ }^{*} p<0.05$ compared to control region. ${ }^{\sharp} p<0.05$ compared to control.

$11 \mathrm{~mm}$ for meningioma. Cerebral angiography was also performed.

After the first blood flow measurement with XeCT, $200 \mathrm{ml}$ of $10 \%$ glycerol was infused for 15 minutes. The second measurement was carried out immediately after the administration of glycerol. Data were analyzed using the t-test. Statistical significance was set at $\mathrm{p}<0.05$.

\section{Results}

Table 1 shows the cerebral blood flow in the various ROIs. There were no significant difference between the blood flows in the whole hemispheres of the tumor and non-tumor sides in cases of glioma or meningioma. The tumor part of cases of glioma was significantly hypoperfused $(p<0.05)$. In contrast, the tumor part of meningioma was significantly hyperperfused $(\mathrm{p}<0.05)$. The peritumoral edema of both glioma and meningioma was hypoperfused $(\mathrm{p}<0.05)$.

The relative values of blood flow were calculated as blood flow after/before the administration of glycerol $\times 100 \%$. Table 1 shows the relative values of blood flow in the various regions. Decreased blood flow was only observed in the tumor part of glioma cases. Increased rates of blood flow after the administration of glycerol in the tumor part and the peritumoral edema of meningioma were relatively low, compared with the control. However, there were no significant differences.

Cerebral angiography showed cases of glioma were hypervascular in seven, normal in seven, and hypovascular in three, and cases of meningioma were hypervascular in 12 and normal in three.

\section{Representative Cases}

Case 1: A 15-year-old female was admitted under a diagnosis of glioblastoma multiforme based on CT.
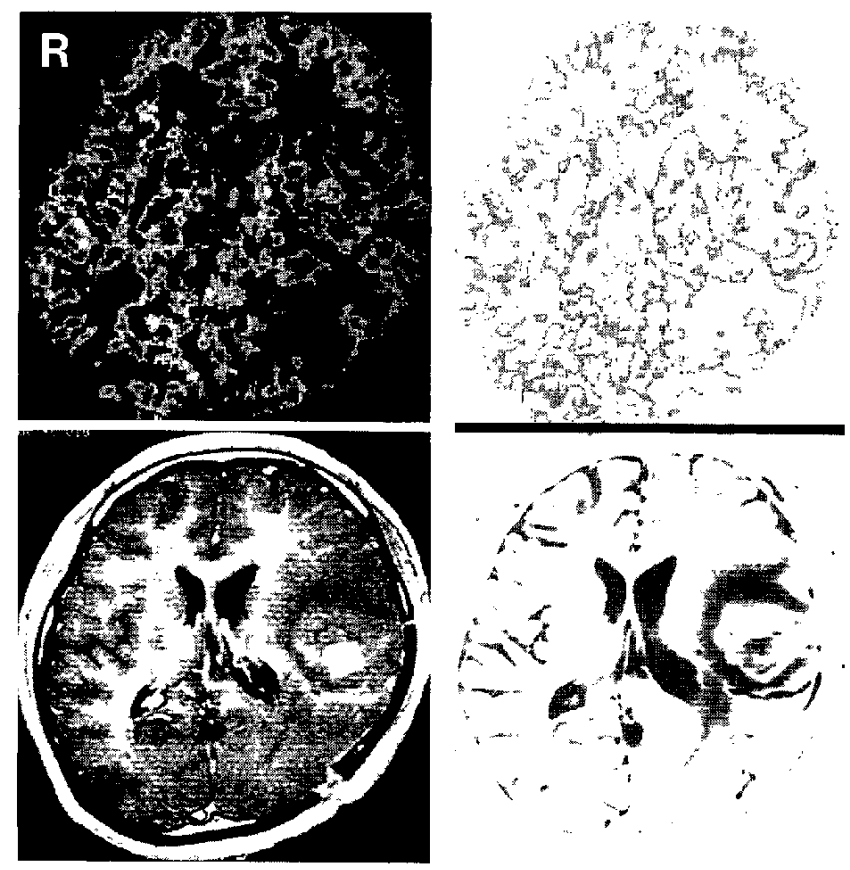

Fig. 1 Case 1. A 15-year-old female with glioblastoma multiforme. Blood flow image (upper left) showing the tumor part and the peritumoral edema were hypoperfused, and after the administration of glycerol (upper right) decreased blood flow only in the tumor part. $T_{1}$-weighted magnetic resonance (MR) image with gadoliniumdiethylenetriaminepenta-acetic acid (lower left) and $\mathrm{T}_{2}$-weighted MR image (lower right).

Neurological examination revealed right hemiparesis and aphasia. Cerebral angiography showed a hypervascular tumor. The histology was confirmed by biopsy. Xe-CT was then carried out (Fig. 1). The blood flow was $34.5 \mathrm{ml} / 100 \mathrm{~g} / \mathrm{min}$ in the tumor, 25.6 $\mathrm{ml} / 100 \mathrm{~g} / \mathrm{min}$ in the peritumoral edema, and $50.0 \mathrm{ml} /$ $100 \mathrm{~g} / \mathrm{min}$ in the control region. The tumor and the 


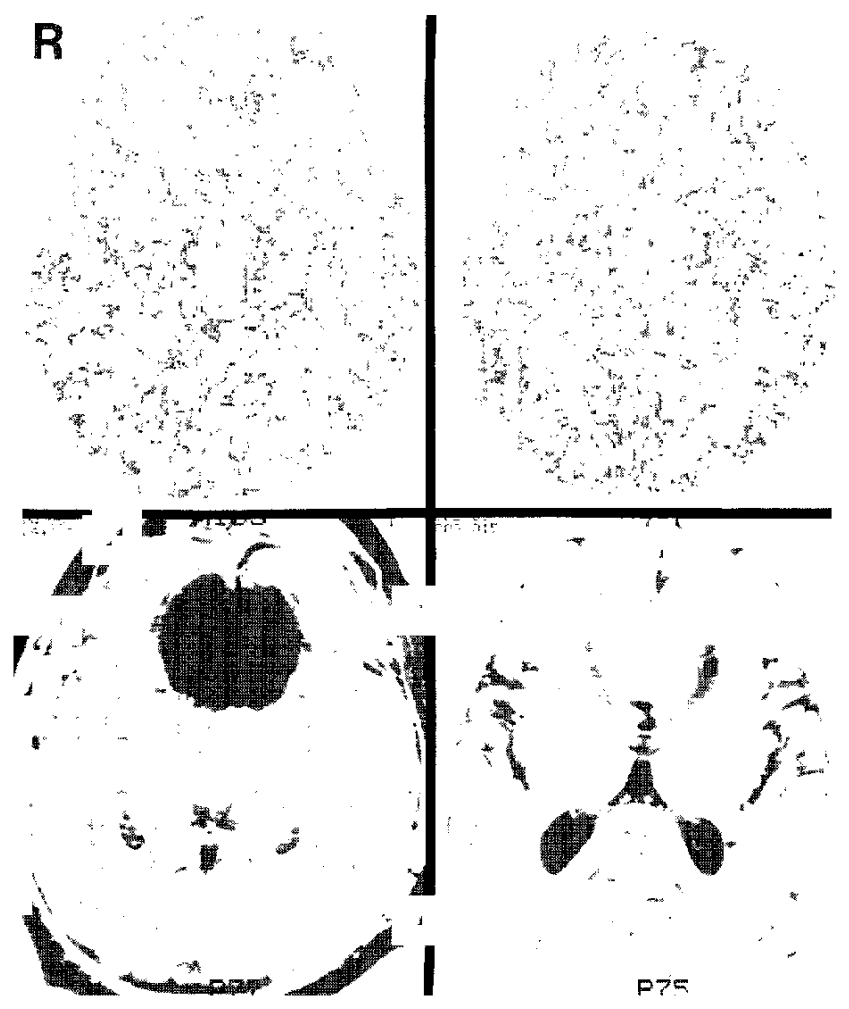

Fig. 2 Case 2. A 60-year-old male with meningioma. Blood flow image (upper left) showing blood flow in the tumor was hyperperfused, and after the administration of glycerol (upper right) increased blood flow in all regions. $T_{1}$-weighted magnetic resonance (MR) image with gadoliniumdiethylenetriaminepenta-acetic acid (lower left) and $\mathrm{T}_{2}$-weighted MR image (lower right).

peritumoral edema were hypoperfused. The relative values of blood flow after the administration of glycerol were $94.7 \%$ in the tumor, $126.9 \%$ in the peritumoral edema, and $120.8 \%$ in the control. Blood flow decreased only in the tumor part.

Case 2: A 60-year-old male noted bilateral visual field disturbance. Neurological examination revealed bitemporal hemianopsia. MR imaging showed a mass homogeneously enhanced with Gd-DTPA in the tuberculum sellae (Fig. 2). Cerebral angiography showed a hypervascular tumor. Xe-CT revealed that the blood flow was $74.1 \mathrm{ml} / 100 \mathrm{~g} / \mathrm{min}$ in the tumor and $30.4 \mathrm{ml} / 100 \mathrm{~g} / \mathrm{min}$ in the peritumoral edema. Blood flow in the control region was not measured because the tumor was located on the midline. The tumor was hyperperfused. The relative values of blood flow after the administration of glycerol were $115.3 \%$ in the tumor and $112.9 \%$ in the peritumoral edema. Total removal was performed and the histolo- gy was confirmed to be meningothelial meningioma.

\section{Discussion}

Blood flow in brain tumors varies. The mean tumor blood flow was less than that in the corresponding region of the contralateral hemisphere in CD Fischer rats with RT-9 brain tumor, but may be related to tumor size, location, and presence of necrosis. ${ }^{2)}$ Blood flow may be influenced by cerebral perfusion pressure (CPP), but not tumor type or grade, as the CPP depends on the tumor origin. ${ }^{6)}$ In our study, we classified the tumors into two groups by origin. Glioma which is an intraaxial tumor, and meningioma which is an extraaxial tumor. Grade was not considered. We found that glioma was hypoperfused and meningioma was hyperperfused. Previous studies have measured blood flow in brain tumors and found that meningioma was hyperperfused and metastatic tumor was hypoperfused, ${ }^{5]}$ and both gliomas and metastatic tumors were hypoperfused. ${ }^{7}$ These differences may not be due to tumor type or grade, but to the tumor origin. Our cerebral angiography findings showed that vascularity was not involved with blood flow. Blood flow is mainly affected by $C P P,{ }^{6]}$ and is not necessarily related to angiography findings. Peritumoral edema occurs in the white matter of glioma and in the subarachnoid space of meningioma, and does not interfere with blood flow or flow regulation as long as intracranial pressure is normal. ${ }^{3)}$ We found peritumoral edema was hypoperfused in both glioma and meningioma cases. Previously, peritumoral edema was found to be hypoperfused ${ }^{5,10)}$ We did not measure intracranial pressure, but this was likely to be high. There were no difference in whole hemispheric blood flow between the tumor side and non-tumor side in both glioma and meningioma cases. Local blood flow changes did not affect global hemisphere, suggest ing blood flow redistribution in the brain.

The effect of glycerol on blood flow in brain tumors is less well known. Blood flo-vs in all regions except the tumor are increased after the administration of glycerol, whereas blood flow in the tumor does not change. ${ }^{10]}$ Measurement of blood flow in hemispheres including meningioma following the administration of glycerol increased by about $20 \%$ in all regions. ${ }^{4)}$ In our study, blood flows in all regions except for glioma increased after the administration of glycerol. Blood flow in the tumor part of glioma cases decreased. The blood brain barrier (BBB) in glioma is gradually destroyed with proliferating pinocytic vesicles and fewer strands of the tight junction according to the progression of tumor malignancy, ${ }^{8)}$ but the integrity of the $\mathrm{BBB}$ is 
not known. Proliferating pinocytic vesicles also occur in meningioma. ${ }^{9]}$ Tumor malignancy cannot be discriminated only by the enhancement due to contrast media in CT or MR imaging. ${ }^{13}$ Presumably fewer strands of the tight junction are involved in prevention of improvement of blood flow by glycerol in the tumor. We found that the blood flow in peritumoral edema increased after the administration of glycerol, presumably caused by steal of blood flow from the tumor to the peritumoral brain tissue. The tumor part of meningioma cases was hyperperfused. Glycerol affected both the tumor and the peritumoral edema. The increases in blood flow in the tumor and the peritumoral edema in meningioma cases after the administration of glycerol were relatively low compared with that of the control region. The ultrastructure of tumor vessels in glioma are similar to normal vessels except for proliferating pinocytic vesicles and fewer strands of the tight junction. No fenestration is found. ${ }^{8)}$ In contrast, the characteristic findings of tumor vessels in meningioma are thinning of endothelium and many fenestrations, short and open tight junctions. Pinocytic vesicles are also observed. ${ }^{9)}$ The strands of the tight junction, with or without fenestration, which form the BBB might be responsible for the difference in response to glycerol. Glycerol affects various brain tumors in different ways. More study of glycerol treatment in patients with brain tumors is needed to assess the ultrastructural function.

\section{References}

1) Agnoli AL, Herrmann S: Contrast medium assisted nuclear magnetic resonance tomography. Signal intensity of gliomatous tumors. Rontgenblatter 43: 335340, 1990

2) Blasberg $R$, Molnar $P$, Horowitz $M$, Kornblith $P$, Pleasants R, Fenstermacher J: Regional blood flow in RT-9 brain tumors. J Neurosurg 58: 863-873, 1983

3) Hossmann KA, Bloink M, Wilmes F, Wechsler W: Experimental peritumoral edema of the cat brain. Adv Neurol 8: 323-340, 1980

4) Ishikawa M, Kikuchi H, Nagata I, Yamagata S, Taki W, Kobayashi A, Yonekura Y, Nishizawa S: [The effect of glycerol on regional cerebral blood flow, blood volume and oxygen metabolism]. No Shinkei Geka 17: 635-640, 1989 (Jpn, with Eng abstract)

5) Kida Y, Ishiguri H, Ichimi K, Kobayashi T: [Evaluation of the regional blood flow in and around brain tumors by means of Xe-enhanced CT]. CT Kenkyu 14: 25-31, 1992 (Jpn, with Eng abstract)

6) Lammetsma AA, Wise RJ, Jones T: In vivo measurements of regional cerebral blood flow and blood volume in patients with brain tumors using positron emission tomography. Acta Neurochir (Wien) 69: 1-2, $5-13,1983$
7) Nakamura O, Nomura K, Segawa H, Takakura K, Nakagome T, Yoshimasu N, Ueda $Y$, Kimura $K$, Nagai M: [rCBF in brain tumors as measured by $\mathrm{Xe}-$ non enhanced CT]. No Shinkei Geka 13: 37-43, 1985 (Jpn, with Eng abstract)

8) Shibata $S$, Fukushima $M$, Inoue $M$, Tsutsumi $K$, Mori $\mathrm{K}$ : [Ultrastructure of capillary permeability in human brain tumors -Part 1: Glioma associated with cerebral edema (low density area)-]. No Shinkei Geka 13: 275-281, 1985 (Jpn, with Eng abstract)

9) Shibata S, Fukushima M, Mori K: [Ultrastructure of capillary permeability in human brain tumors - Part 4. Cerebral edema associated with meningioma- - . No Shinkei Geka 14: 613-618, 1986 (Jpn, with Eng abstract)

10) Shimoda $M$, Kawamata F, Yamamoto $M$, Ohsuga $H$, Hidaka M, Oda S, Shibuya N, Yamamoto I, Sato O: [The evaluation of cerebral hemodynamics in patients with intracranial tumors by stable xenon CT: The effect of glycerol administration on regional blood flow]. CT Kenkyu 11: 161-168, 1989 (Jpn, with Eng abstract]

Address reprint requests to: M. Niwa, M.D., Department of Neurosurgery, Nakatsugawa Municipal General Hospital, 1522-1 Komamba, Nakatsugawa, Gifu 508, Japan.

\section{Commentary}

In this study the authors have nicely confirmed previous findings of reduced blood flow around tumors of both types, with added intratumoral hypoperfusion in gliomas but hyperperfusion in meningiomas. After glycerol, flow fell even further, and significantly, in gliomas, rose further but not significantly in meningiomas, and increased to an extent in the peritumoral area around both tumor types. A possible explanation is offered for the different responses of the two types of tumor, based on anatomical differences in the tight junctions affecting blood-brain barrier function. Could the difference somehow also have something to do with the much larger area of edema around gliomas, where the maximum was $28 \mathrm{~mm}$ compared with $11 \mathrm{~mm}$ around meningiomas?

It is interesting that the only actual decrease of flow was the tumor itself in glioma cases. As noted, this may be evidence of a type of steal phenomenon, with the tumor vessels being unable to dilate and increase their flow. Perhaps this is connected with the poor vascularity in gliomas, and part of the explanation for the center of gliomas, especially glioblastomas, often becoming necrotic.

Another point of interest is that the increase in the peritumoral region of gliomas was on average propor- 
tionately more than the increase in the tumor hemisphere as a whole. This raises the question of whether there could in some circumstances also be a steal, at least relative, from the area around into the immediate peritumoral area.

Nicholas W. C. DorSCH, M.D., F.R.C.S., F.R.A.C.S. Department of Neurosurgery Westmead Hospital Sydney, Australia

Glycerol is a popular and useful material in the neurosurgical field. However, the mechanism of its effect on brain tumors is not yet well understood. In this study, the authors analyzed the effect of glycerol on blood flow in brain tumors, meningioma and glioma. They found a decrease of blood flow in the tumor part of glioma and an increase in the peritumoral edema after the intravenous administration of glycerol.

The mechanism of this effect is explained by the steal of blood flow from the tumor to the peritumoral tissue. The authors' hypothesis sounds attractive but its basis is insufficient. More detailed observation of blood flow changes are needed, particularly in relation with time elapse. The authors also state that the blood brain barrier in tumors might be responsible for the different response between glioma and meningioma. To confirm such a hypothesis, much more investigation of many kinds of brain tumors will be necessary. Also, the difference between benign and malignant tumors should also be investigated.

Shigeharu SUZUKI, M.D. Department of Neurosurgery Hirosaki University School of Medicine Hirosaki, Aomori, Japan

Niwa et al. used stable xenon-enhanced computed tomography to measure cerebral and tumor blood flow before and after glycerol infusion in 32 patients. They found that the blood flow in the glioma significantly decreased after administration of glycerol. It has been generally accepted that a hypertonic solution like glycerol increases the cerebral blood flow. We believe that this mechanism is due to the decrease in blood viscosity resulting from hemodilution or the increase in perfusion pressure caused by the decompressive effect. It is noteworthy that the blood flow in the tumor part of glioma decreases in response to glycerol infusion. The authors speculated that this occurred by blood steal from the tumor to the cerebral tissue, although they did not explain clearly why the blood was stolen in this direction. However, we have to consider their new and interesting results in this study.

Mamoru TANEDA, M.D. Department of Neurosurgery Kinki University School of Medicine Osaka-sayama, Osaka, Japan

In this article, the authors demonstrated that blood flow in meningioma is higher than blood flow in normal brain tissue whereas blood flow in areas of peritumoral edema and glioma is lower. The administration of glycerol can eventually increase blood flow in meningioma, in areas with peritumoral edema as well as in normal brain tissue, but not in glioma. This effect of blood flow increase in normal brain of two studied groups $(25.1 \%$ and $21.9 \%$, respectively) and peritumoral edema of glioma (19.7\%) is much higher than in meningioma (9.2\%) and its peritumoral edema $(8.8 \%)$. The authors attribute the decrease of flow in glioma after glycerol infusion to the disruption of blood brain barrier (BBB) which causes steal of flow from tumor to the areas of peritumoral edema. In general, the degree of BBB disruption varies with the degree of tumor malignancy. Changes of flow pattern between glioblastoma and astrocytoma in the glioma group or between meningothelial meningioma and malignant meningioma in the meningioma group, however, are not further analyzed in this study. Correlation between the morphological changes in the ultrastructure of $B B B$ of the tumor and peritumoral edema, and the effects of glycerol on tumoral blood flow would be an interesting topic for further study.

Yong-Kwang TU, M.D.

Division of Neurosurgery College of Medicine and University Hospital National Taiwan University Taipei, Taiwan 